\title{
Essential role for ALCAM gene silencing in megakaryocytic differentiation of K562 cells
}

\author{
Fang Tan', Samit Ghosh', Flaubert Mbeunkui ${ }^{2}$, Robert Thomas ${ }^{2}$, Joshua A Weiner ${ }^{3}$, Solomon F Ofori-Acquah ${ }^{*}$
}

\begin{abstract}
Background: Activated leukocyte cell adhesion molecule (ALCAM/CD166) is expressed by hematopoietic stem cells. However, its role in hematopoietic differentiation has not previously been defined.

Results: In this study, we show that ALCAM expression is silenced in erythromegakaryocytic progenitor cell lines. In agreement with this finding, the ALCAM promoter is occupied by GATA-1 in vivo, and a cognate motif at -850 inhibited promoter activity in K562 and MEG-01 cells. Gain-of-function studies showed that ALCAM clusters K562 cells in a process that requires PKC. Induction of megakaryocytic differentiation in K562 clones expressing ALCAM activated PKC- $\delta$ and triggered apoptosis.

Conclusions: There is a lineage-specific silencing of ALCAM in bi-potential erythromegakaryocytic progenitor cell lines. Marked apoptosis of ALCAM-expressing K562 clones treated with PMA suggests that aberrant ALCAM expression in erythromegakaryocytic progenitors may contribute to megakaryocytopenia.
\end{abstract}

\section{Background}

Hematopoiesis is controlled by interactions between hematopoietic stem cells and their microenvironment. These interactions influence retention of stem cells in specific niches, and stem and progenitor cell expansion, lineage divergence and differentiation [1]. Adhesion molecules are major regulators of cell-cell interactions and they influence multiple aspects of hematopoiesis [1-4]. Indeed, antibodies against various adhesion molecules including VLA-4 and VCAM-1 inhibit the ability of hematopoietic stem cells to populate the bone marrow of irradiated mice [5], and gene knock-out studies of integrins have shown their critical role in homing and colonization of late-stage primary hematopoietic organs such as the embryonic liver [6,7]. More recently, $\mathrm{N}$-cadherin expression has been implicated in retention of hematopoietic stem cells in the bone marrow niche [8-10] although this claim is not supported by other studies [11]. In contrast to their role in homing, our understanding of adhesion molecule biology in lineage commitment and differentiation is poorly defined.

\footnotetext{
* Correspondence: soforia@emory.edu

'Department of Pediatrics, Aflac Cancer Center and Blood Disorders Service, Emory University School of Medicine, Atlanta, GA 30322, USA

Full list of author information is available at the end of the article
}

Hematopoietic cell antigen, also known as activated leukocyte cell adhesion molecule (ALCAM/CD166), is a member of the immunoglobulin super-family. It is expressed on the surface of the most primitive hematopoietic cells in human fetal liver and fetal and adult bone marrow [12]. Other studies have found ALCAM expression on subsets of stromal cells in the para-aortic mesoderm and other primary sites of hematopoiesis in the human embryo [13]. ALCAM-mediated interactions are important during neural development [14], maturation of hematopoietic stem cells in blood forming tissues [12,15], immune responses [16] and in tumor progression [17]. Anti-ALCAM antibodies inhibit myeloid colony formation in vitro by mechanism that remains unknown [18]. We showed previously that ALCAM is involved in transmigration of monocytes across endothelial monolayers [19]. More recent in vivo studies have shown that ALCAM is essential for monocyte migration across the blood-brain barrier [20]. Other studies indicate the interaction of ALCAM on dendritic cells with the T-cell ligand CD6 is required for optimal $\mathrm{T}$-cell activation [21]. While these studies highlight ALCAM's role in mature and activated leukocyte cell biology, there is currently no information on ALCAM's role in hematopoietic progenitor cell biology.
C Biomed Central

C 2010 Tan et al; licensee BioMed Central Ltd. This is an Open Access article distributed under the terms of the Creative Commons Attribution License (http://creativecommons.org/licenses/by/2.0), which permits unrestricted use, distribution, and reproduction in any medium, provided the original work is properly cited. 
In this study, we examined ALCAM expression in human hematopoietic cell lines. The ALCAM gene was cloned and functionally characterized in K562 cell lines. The influence of ALCAM on megakaryocytic differentiation of K562 cells was investigated.

\section{Results}

Lineage-specific expression of ALCAM in hematopoietic progenitor cell lines

Previous studies have documented ALCAM surface expression on hematopoietic stem and progenitor cells. In this study, we quantified ALCAM mRNA expression in multiple human hematopoietic progenitor cell lines of myeloid, lymphoid, erythroid, and megakaryocytic lineages by real-time quantitative PCR. ALCAM mRNA was most abundant in THP-1 monocytes, at a level 2-fold higher than in HL-60 cells, and 8-fold higher than in U-937 and Jurkat cells (Figure 1A). No ALCAM transcripts were however detected in K562 and MEG-01 cells (Figure 1A). This expression pattern was confirmed at the protein level as none of the erythromegakaryocytic progenitor cell lines (K562, MEG-01) expressed ALCAM, while ALCAM protein was abundant in THP-1 monocytes (Figure 1B).

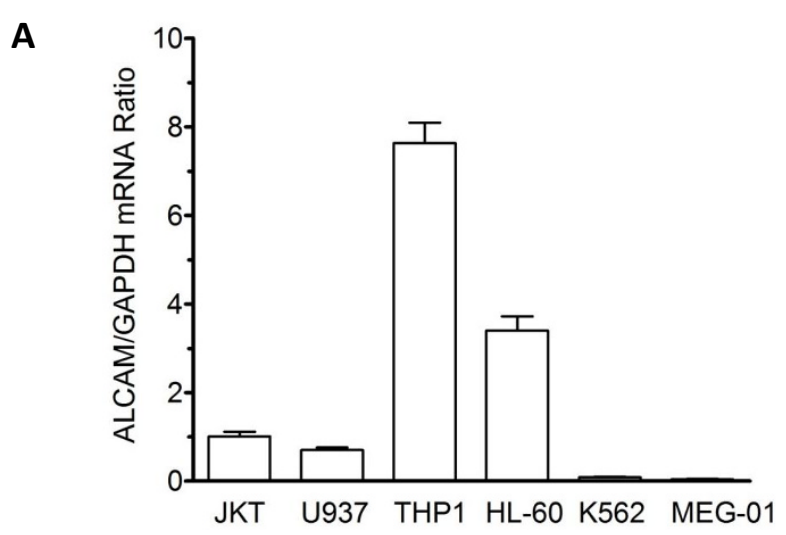

B

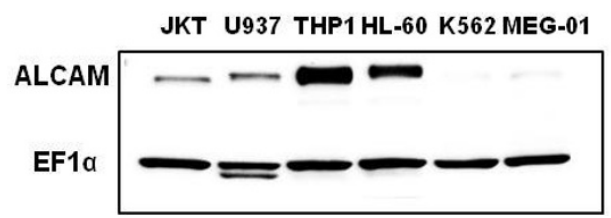

Figure 1 ALCAM expression in hematopoietic progenitor cell lines. A) Total RNA was isolated from hematopoietic cells and ALCAM mRNA quantified by quantitative RT-PCR. GAPDH was used as invariant control in the experiment. Data shown is the mean of three analyses each in triplicates. B) Whole cell lysates $(15 \mu \mathrm{g})$ from indicated cells were blotted for ALCAM protein by western blot analysis and protein loading verified by analyzing the same filters for EF-1 $\alpha$ expression.

\section{A negative GATA-1 binding element in the ALCAM promoter}

Thus far, we had identified an expression pattern for ALCAM that was consistent with regulation of the ALCAM gene by erythroid and megakaryocytic transcription factors. To investigate this idea, multiple fragments of the ALCAM 5'-flanking region was cloned, sequenced and its activity analyzed in K562 and MEG01 cells. Activity of the p650 construct was on average 40-fold higher compared to pGL3 in both cell types (Figure 2A). Activity of p1000 was significantly lower compared to p800, which suggested the presence of negative regulatory cis element in the interval -800 to -1000 of the ALCAM gene. Sequence analysis using the TRANSFAC 7.0 software identified a GATA-1 motif at -850. Mutation of this cis element increased activity of p1000 by 3 -fold (Figure 2B). To confirm a role for GATA-1, K562 nuclear extracts were used in gel mobility shift assay. A single major protein-DNA complex formed on a -850 ALCAM DNA probe using nuclear extracts from K562 and MEG-01 cells (Figure 2C, lane 2 and data not shown). Formation of this complex was competitively blocked by unlabeled wild-type -850 ALCAM GATA probe indicating the specificity of this cis element in the protein-DNA complex (lanes 3-5), and by anti-GATA-1 antibody (lane 6), which confirmed that the GATA-1 protein is a component of this protein-DNA complex. On the contrary, increasing molar excess of an unlabeled mutant -850 ALCAM probe failed to compete for binding (lanes 7-9). Finally, we prepared chromatin from K562 cells to determine whether GATA-1 occupied the ALCAM promoter in vivo. Results of ChIP assay experiments confirmed that GATA-1 binds to the endogenous promoter in $\mathrm{K} 562$ cells (Figure 2D). Collectively, these findings suggest that ALCAM silencing is part of the mechanism of GATA-1 mediated megakaryocytic differentiation.

\section{Ectopic ALCAM expression clusters K562 cells in a PKC- dependant manner}

To determine the functional relevance of ALCAM silencing, we established clones of K562 stably expressing ALCAM linked to a green florescent protein (K562ALCAM) or a GFP vector (K562). ALCAM was abundantly expressed in these stable K562 clones while it was absent in the control clones expressing GFP (Figure $3 \mathrm{~A})$. Live-cell imaging showed that ALCAM was recruited to sites of cell-cell contact in the K562ALCAM clones whereas GFP remained distributed in the cytosol in the control K562-GFP clones (Figure 3B). Unlike parental K562 and K562-GFP clones, the K562ALCAM clones consistently formed large clusters in culture, spontaneously. To test whether ALCAM is responsible for this behavior, a function blocking anti- 
A

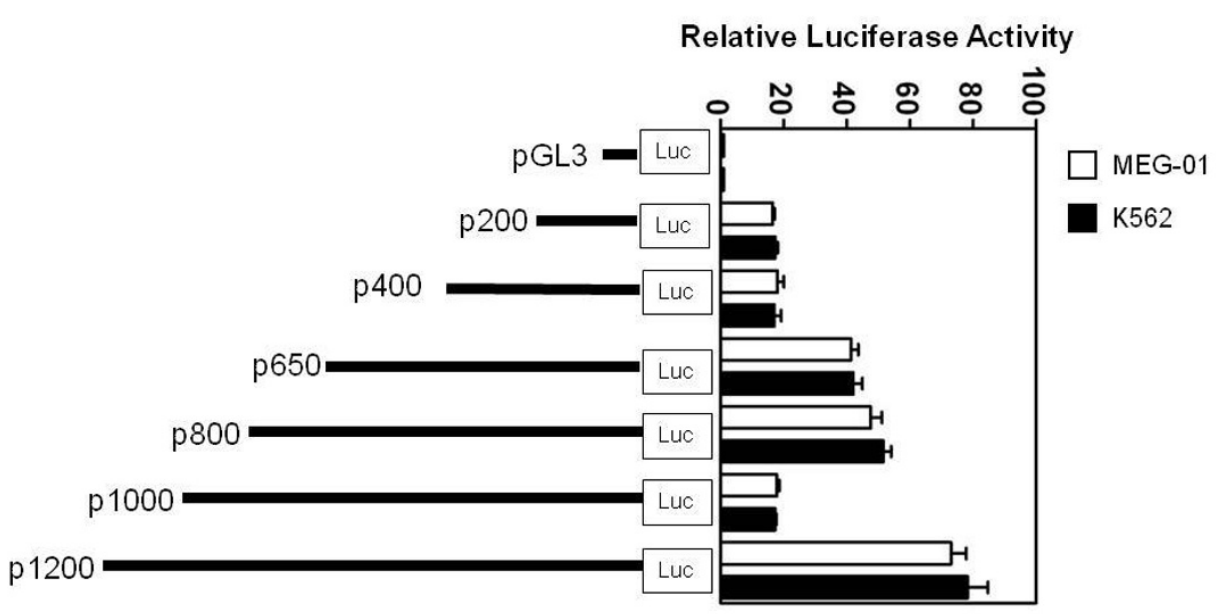

B

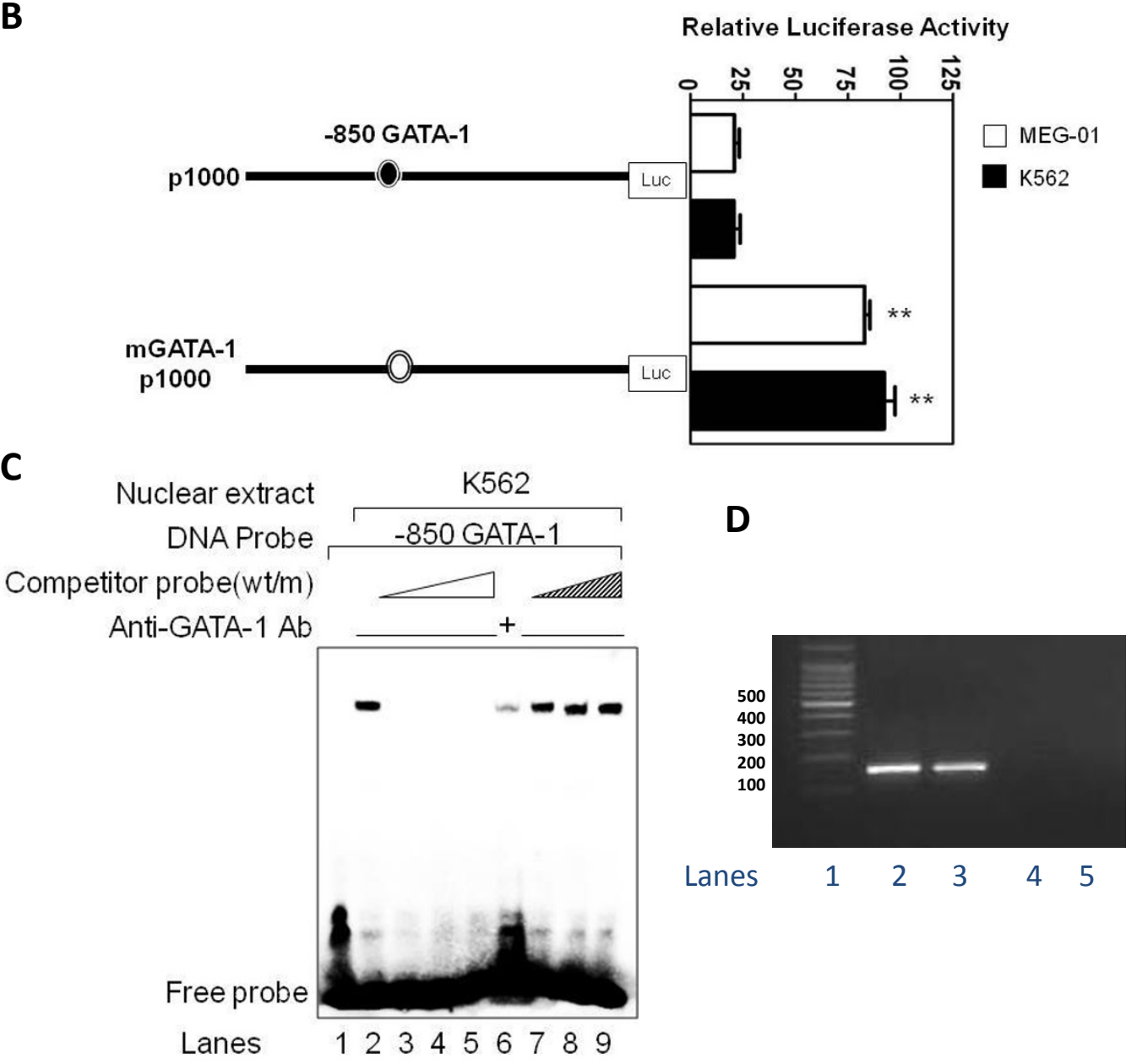

C

Figure 2 A functional GATA-1 element in the proximal ALCAM promoter. A) Schematic diagram of reporter constructs consisting of truncated ALCAM promoter fragments driving expression of pGL3. Histogram shows the relative luciferase activity for each construct in K562 and MEG-01 cells. B) Reporter constructs showing the presence of wild-type (filled circle) or mutant (open circle) GATA-1 cis element at -850 in the human ALCAM promoter. Histogram shows the relative luciferase activity for each construct in K562 and MEG-01 cells. C) Electrophoretic mobility shift assay showing protein-DNA complexes in experiments performed with the -850 GATA probe and no nuclear extracts (lane 1), or with K562 nuclear extracts alone (lane 2), and in the presence of molar excess of unlabeled wild-type probe (lanes 3-5), anti-GATA1 antibody (lane 6) and unlabeled mutant -850 GATA1 probe (lanes 7-9). D) Ethidium bromide stained gel of ChIP assay PCR products showing a 151 bp product for input DNA (lane 2), and products from chromatin immunoprecipitations performed with anti-GATA1 antibody (lane 3), IgG (lane 4) and without chromatin (lane 5). Lane 1 contains DNA size marker. 


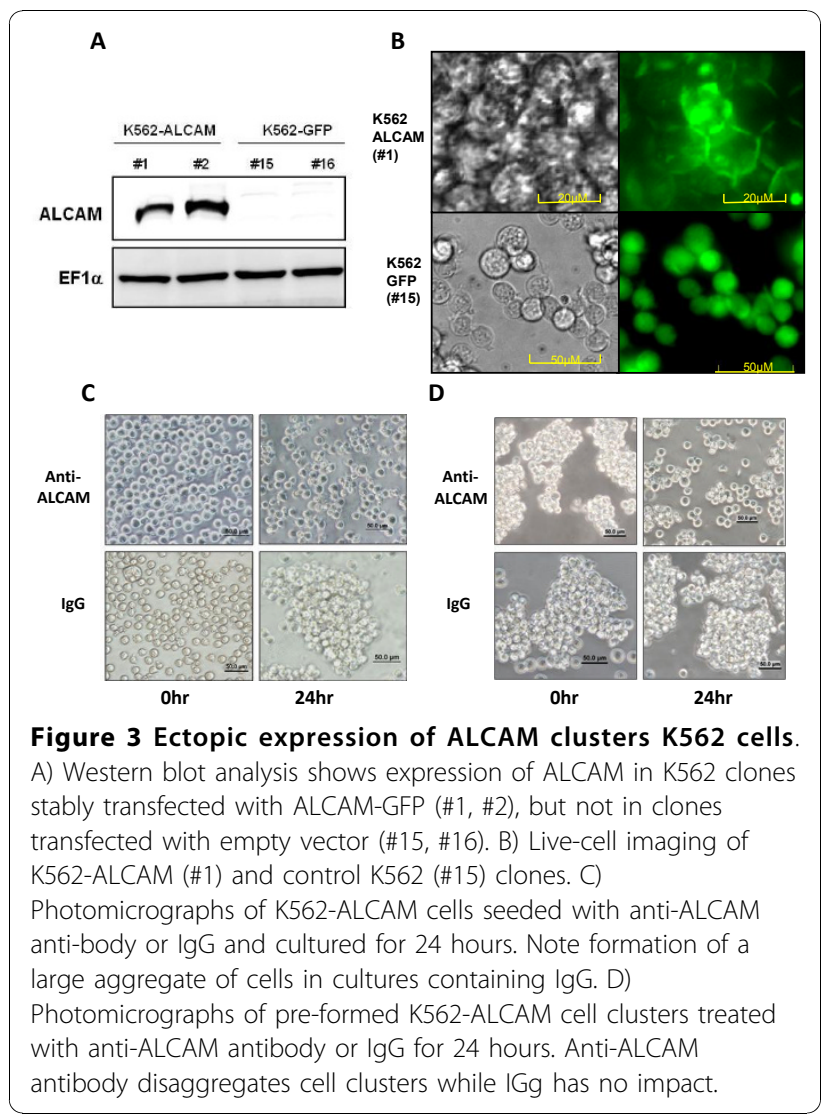

ALCAM antibody that we and others have shown previously to inhibit ALCAM-mediated homotypic adhesion was added to cultures. Anti-ALCAM antibody $(10 \mu \mathrm{g} / \mathrm{ml})$ prevented cell clustering whereas an isotypematched IgG had no effect (Figure 3C). Moreover, anti-ALCAM antibody disaggregated pre-formed K562ALCAM clusters while isotype-matched IgG had no impact (Figure 3D). PKC- $\alpha$ has previously been implicated in ALCAM-mediated cytoskeletal alterations in K562 cells, therefore we tested whether this mediator was involved in ALCAM-induced K562 clustering. Inhibition of PKC dose-dependently blocked clustering of K562-ALCAM cells, while DMSO had no impact on this behavior (Figure 4A). Moreover, the pan-PKC inhibitor chelerythrine chloride dose-dependently disaggregated previously formed large aggregates of K562ALCAM cells (Figure 4B). These data indicate that PKC contributes to the ALCAM-mediated aggregation of hematopoietic cells.

To analyze the PKC mechanisms involved in ALCAMmediated cell clustering, we examined expression of PKC isoforms by qRT-PCR. There was significantly increased expression of PKC- $\alpha$, but not of PKC- $\delta,-\varepsilon$ or $-\theta$ in these cells at baseline. The level of PKC- $\alpha$ mRNA was 3 -fold higher in K562-ALCAM clones than in

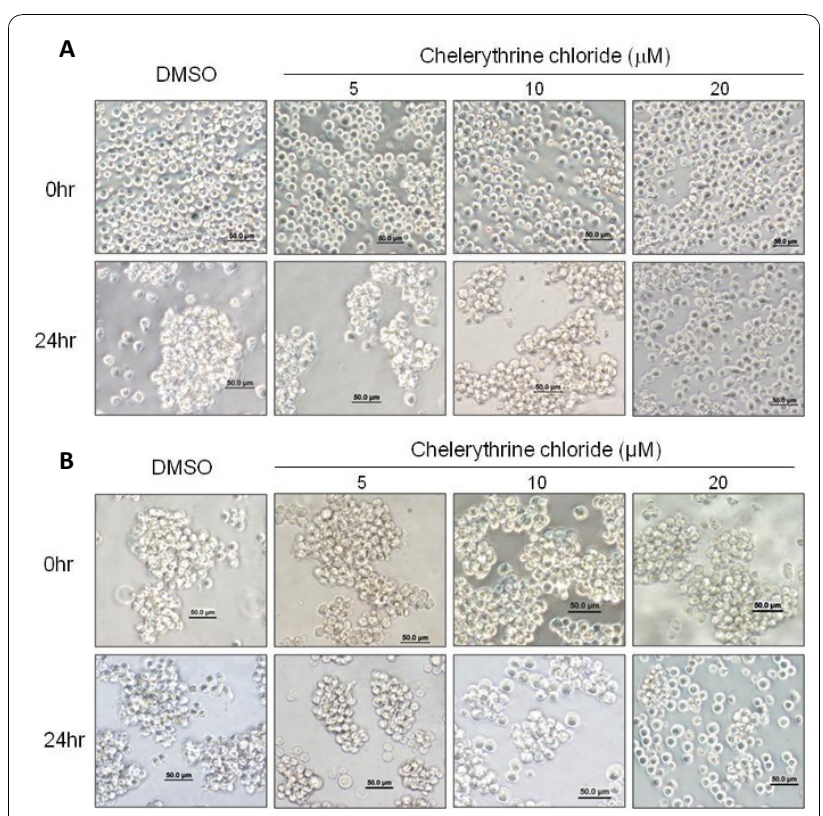

Figure 4 ALCAM-induced clustering of K562 cells is PKCdependant. A) Photomicrographs of K562-ALCAM single cell suspensions treated with a concentration $(5-20 \mu \mathrm{M})$ range of chelerythrine chloride or DMSO (vehicle) at the time of seeding $(0$ hr) and 24 hours later. Chelerythrine chloride dose-dependently inhibits the formation of clusters of K562-ALCAM. B) Photomicrographs of preformed K562-ALCAM clusters treated with a concentration $(5-20 \mu \mathrm{M})$ range of chelerythrine chloride or DMSO (vehicle). Chelerythrine chloride dose-dependently disaggregates pre-formed clusters. Cultures were photographed using Olympus IX50 microscope (Olympus Optical Co. Ltd. Japan).

control cells (Figure 5A). The pan-PKC activator PMA significantly increased mRNA expression of three isoforms $(\alpha, \varepsilon$ and $\theta)$ in both K562-ALCAM and the control K562-GFP cells (Figure 5A). PKC- $\delta$ mRNA expression increased significantly in the K562-ALCAM clones, but not in control cells. Next, we examined steady-state and PMA-induced levels and activation of PKC- $\alpha$ and PKC- $\delta$. The level of PKC- $\alpha$ protein was increased in K562-ALCAM clones, at steady-state and following PMA treatment, however PMA did not increase activation of PKC- $\alpha$ (Figure $5 \mathrm{~B}, \mathrm{C}$ ). On the contrary, PMA activated PKC- $\delta$ in the K562-ALCAM clones, while the activation status in control cells remained unchanged (Figure 5B, C).

\section{Altered erythromegakaryocytic gene expression in K562-ALCAM clones}

K562 is an established model for megakaryocytic differentiation in a process that is PKC- $\varepsilon$ dependent $[22,23]$. Thus our data showing that ectopic expression of ALCAM in K562 cells increased expression and activation of PKC- $\alpha$, suggested that the normal megakaryocytic pathway may be altered in these clones. Transcripts 
A
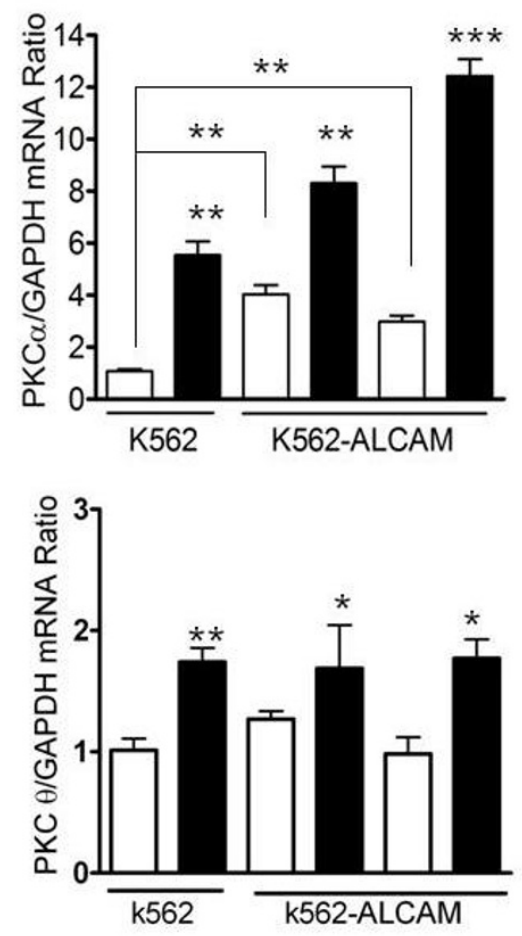

B
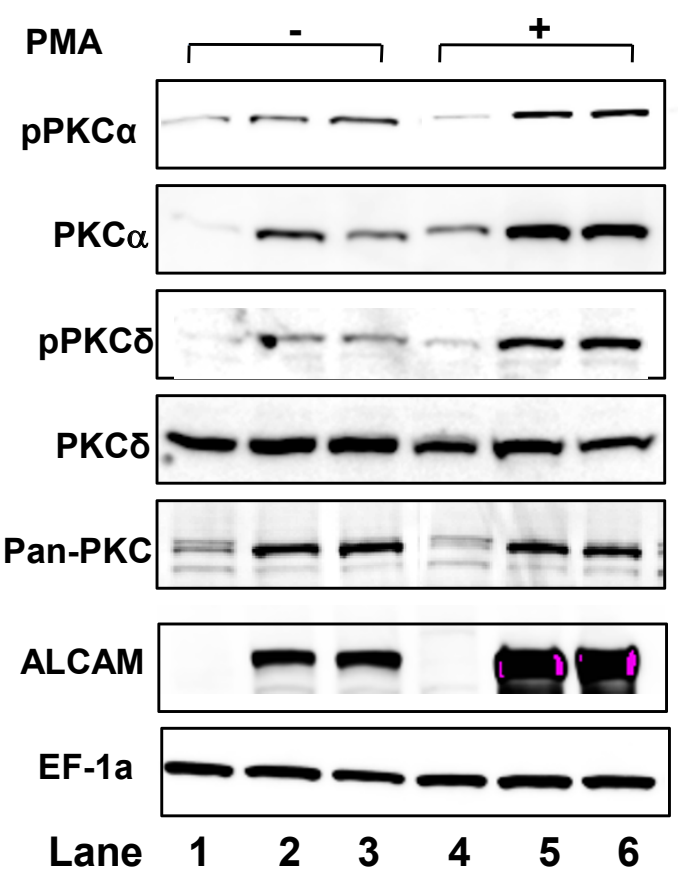
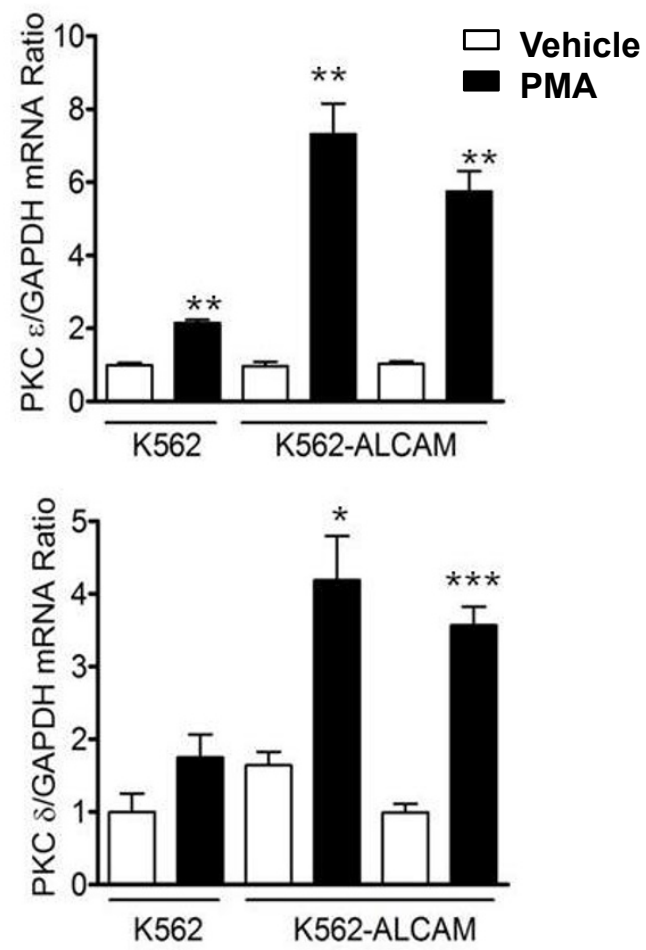

C
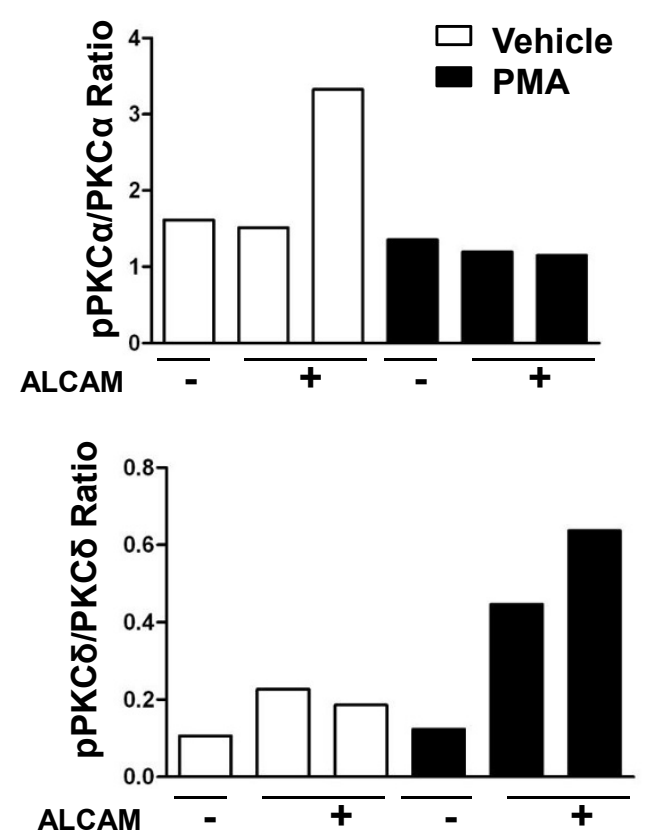

Figure 5 ALCAM alters expression of PKC in K562 cells. A) Quantification of PKC mRNA level in K562-ALCAM (\#1, \#2) and K562 (\#15) clones at steady-state (vehicle) and following treatment with $20 \mathrm{nM}$ PMA for $48 \mathrm{~h}$. B) Western blot analysis showing expression of the indicated proteins in control K562 clone \#15 (lanes 1, 4), ALCAM-expressing clones \#1(lane 2, 5) and \#2 (lanes 3, 6) at steady-state (-) and following treatment with PMA (+). C) Quantification of PKC $\alpha$ and PKC $\delta$ activation. 


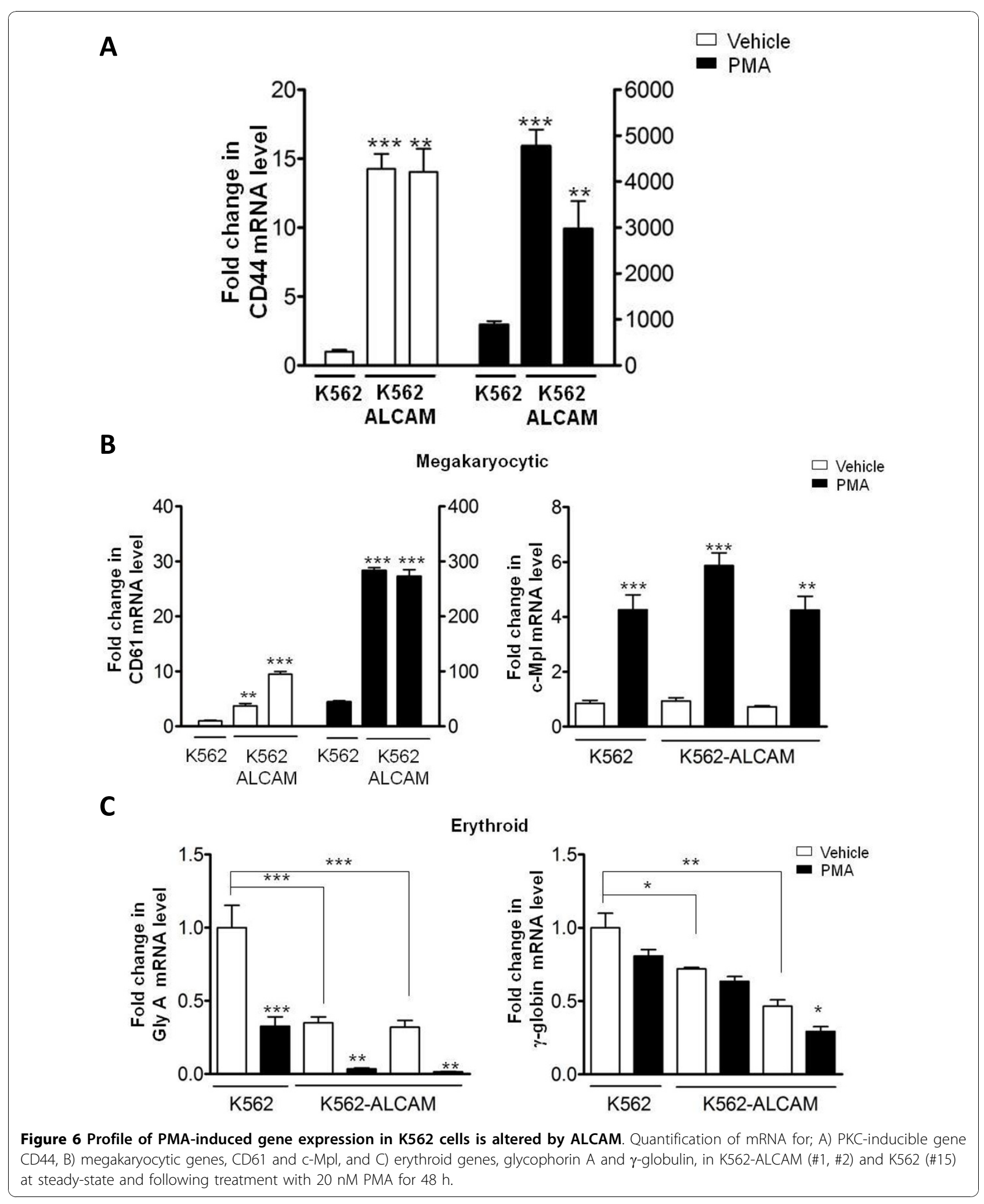


for the PMA-inducible gene CD44 was greater than 10-fold higher in the K562-ALCAM clones compared to control K562 cells at baseline, while PMA increased its expression 3 to 5 -fold higher in K562-ALCAM clones compared to control K562 cells (Figure 6A). With respect to hematopoietic genes, transcripts for the megakaryocytic marker CD61 was 3-10 fold higher in K562-ALCAM clones in the absence of PMA although this was not matched by a comparable increase in cMpl expression (Figure 6B). The level of mRNA for the erythroid genes glycophorin A and $\gamma$-globin was significantly reduced in K562-ALCAM clones compared to K652 control at baseline (Figure 6C). As expected PMA increased mRNA level of megakaryocytic genes and concomitantly decreased erythroid gene expression in the various $\mathrm{K} 562$ clones (Figure $6 \mathrm{~B}, \mathrm{C}$ ). In agreement with our findings at steady-state, $\mathrm{c}-\mathrm{Mpl}$ expression in $\mathrm{K} 562$ ALCAM clones did not increase beyond the level of control K562 cells following induction with PMA. Discordant expression of CD61 and c-Mpl reinforced the notion that megakaryocytic differentiation in the K562ALCAM clones was likely dysregulated.

\section{Apoptosis of K562-ALCAM clones undergoing megakaryocytic differentiation}

K562 cells treated with PMA are well known to display multiple characteristic of megakaryocytic differentiation. To further investigate the impact of ALCAM on K562 cells, we examined cells treated with PMA for the morphological modifications that characterize megakaryocytic differentiation. Cells stained with May-Grunwald-Giemsa revealed that control K562 cells displayed multiple phenotypes consistent with megakaryocytic differentiation, including cell enlargement and extensive vacuolation (Figure 7A, lower panel, arrow). On the contrary, cultures of K562-ALCAM treated with PMA were predominated by amorphous nuclear remnants devoid of recognizable cytoplasm (arrow heads). Other cell types in the K562-ALCAM cultures were markedly smaller with condensed chromatin, and fragmented nucleus consistent with apoptosis (Figure 7A, asterisk). Apoptosis in K562-ALCAM clones was confirmed first, by determining the proportion of cells at the early stage of this process, as defined by surface expression of annexin V. At baseline, the proportion of annexin $\mathrm{V}$-positive cells was similar (10\%) in cultures of $\mathrm{K} 562$ ALCAM and K562 clones. However, treatment of cultures with PMA for 24 hours increased annexin Vpositive cells exclusively among K562-ALCAM cells by 3-fold (Figure 7B). Finally, vehicle and PMA-treated cultures were examined for degradation of DNA using the TUNEL assay. At baseline there was no difference in the percentage of TUNEL-positive cells in either cell type, or in cells treated with DMSO (Figure $7 \mathrm{C}$ and data

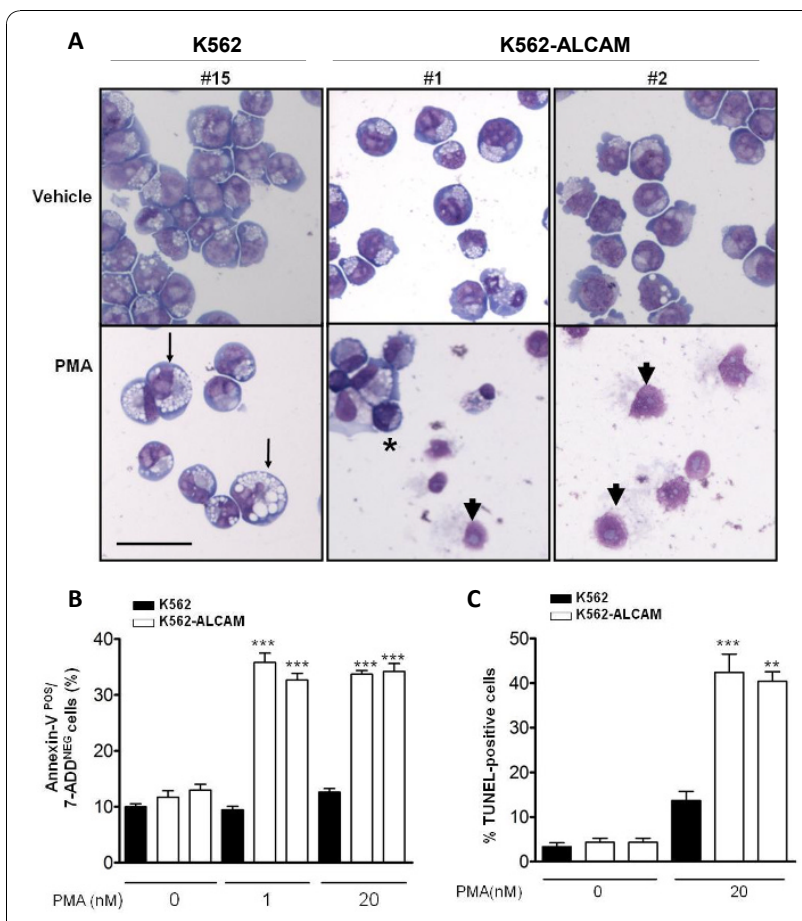

Figure 7 ALCAM promotes apoptosis of K562 cells treated with PMA. A) Photomicrographs of control K562 (\#15) and K562-ALCAM clones (\#1, \#2) stained with May-Grunwald Giemsa, at steady-state (upper panel) or following treatment with 20 nM PMA (lower panel) for 48 hours. PMA caused enlargement and extensive vacuolation in K562 cells (arrow) and nuclear fragments in K562-ALCAM cells (arrow head). B) Apoptosis as determined by the expression of Annexin V in K562 and K562-ALCAM cells at steady-state and following treatment with PMA for 24 hours. C) Apoptosis as determined by the TUNEL assay in K562 and K562-ALCAM cells at steady-state and following treatment with PMA for 48 hours. Scale bar: $50 \mu \mathrm{m}$.

not shown). Treatment with 20 nM PMA for 48 hours increased the percentage of TUNEL-positive cells by an average 4-fold higher in K562-ALCAM compared to K562 cultures.

\section{Discussion}

We report that ALCAM is selectively down-regulated in erythromegakaryocytic progenitor cell lines, and that this phenomenon is essential for survival of K562 cells induced to differentiate towards the megakaryocytic lineage. Our findings suggest that erythromegakaryocytic progenitors that escape ALCAM down regulation may contribute to megakaryocytopenia.

ALCAM was originally thought to be a stage-specific molecule rather than a lineage-specific marker, based on observations that its expression was lost as avian hematopoietic progenitor cells proliferated and differentiated into mature colonies of diverse lineages [24]. More recently, it has become clear that ALCAM is expressed by a diversity of mature leukocytes however its lineage 
identity remained undefined. Data presented in this study show that ALCAM expression is turned off exclusively in progenitor cell lines of the erythromegakaryocytic lineage (Figure 1). Taken together, this suggests that a factor controlling erythromegakaryopoiesis silences ALCAM gene expression. GATA-1 is an excellent candidate for this role given its dominant influence on the survival and differentiation of erythroid and megakaryocytic progenitors [25,26]. Moreover, GATA-1 acts as a gene silencer $[27,28]$ and a lineage-determining factor that suppresses myelomonocytic gene expression while promoting erythroid and megakaryocytic lineage commitment [29]. In support of this paradigm, we found that GATA-1 is abundantly expressed in the K562 and MEG-01 cells used in this study, which lack ALCAM expression, while it is absent in the monocytic cells that abundantly express ALCAM (data not shown). More specifically, we found that there is a GATA-1 cis element in the ALCAM promoter that inhibits promoter activity, and is bound by GATA-1 in vivo, thus providing additional data to support this general paradigm.

As expected, anti-ALCAM antibody inhibited clustering of K562-ALCAM clones, confirming that this phenomenon is dependent on ALCAM adhesion. Corbel et al. [18] used a similar approach to reveal the requirement for ALCAM adhesion in the growth of macrophage, granulocyte and granulocyte/macrophage progenitors. However, anti-ALCAM antibody had no impact on the formation of thromboblast/erythroblast clusters, or growth of erythroid colonies derived from BFU-progenitors [18]. This is in agreement with our findings in wild-type $\mathrm{K} 562$ cells grown in media containing anti-ALCAM antibody (data not shown). We have found that the amount of ALCAM mRNA and protein expressed in the human erythroleukemia cell line HEL is virtually undetectable, similar to what is reported here for K562 and MEG-01 (data not shown). While we cannot exclude the possibility that ALCAM is expressed by immature erythromegakaryocytic precursors, ALCAM silencing in mature precursors would facilitate their segregation from their myeloid counterparts that continue to express ALCAM. In this context apoptosis would be an effective mechanism to ensure lineage fidelity among erythromegakaryocytic precursors that continue to express ALCAM, as demonstrated by this study (Figure 7). That ALCAM expression is pro-apoptotic is contrary to its protective role against apoptosis in breast cancer cells [30]. This apparent discordance is consistent however with the notion that ALCAM is at the crossroads of opposing phenotypes such as adhesion and migration [31]. ALCAM may therefore be involved in influencing the balance between survival and apoptosis, at least, in the hematopoietic system.
Megakaryocytic differentiation is orchestrated by multiple signaling pathways including activation of PKC $\varepsilon$ in both CD34 hematopoietic stem cells and in $\mathrm{K} 562$ cells [32]. The major trigger for this event in $\mathrm{K} 562$ cells is PMA, which activates multiple PKC isoforms. Ectopic expression of ALCAM in K562 cells activated PKC, with functional downstream effects on cell aggregation and gene expression, in the absence of an external agonist (Figure 3, 4 and 5). PKC isoforms have distinctly unique impacts on survival and apoptosis, with the conventional isoforms, particularly PKC- $\alpha$ and PKC- $\beta$ being pro-survival $[33,34]$, the novel isoform, $\mathrm{PKC}-\delta$ is primarily proapoptotic $[35,36]$, whereas $\mathrm{PKC}-\varepsilon$ suppress apoptosis in most studies $[37,38]$. Enhanced expression of PKC- $\alpha$ in K562-ALCAM clones provided an early indication of a potentially different signaling pattern in these clones. PKC- $\delta$ was markedly activated without a comparable activation of the pro-survival PKC- $\alpha$ (Figure 5B, C). This pattern of activation may explain the increased apoptosis of K562-ALCAM clones in response to a signal that normally promotes differentiation towards the megakaryocytic lineage.

\section{Conclusions}

Our data suggest, for the first time, that silencing of ALCAM at the bi-potential erythromegakaryocytic stage may be crucial for cell survival during megakaryocytic differentiation.

\section{Methods}

\section{Chemicals and antibodies}

Phorbol-12-myristate-13-acetate (PMA), Chelerythrine chloride and lipopolysaccharide were purchased from Sigma (St Louis, MO). Monoclonal anti-ALCAM antibody were purchased from Novocatra Laboratories Ltd (Novocatra, Newcastle, UK), anti-PKC isotype-specific antibodies, anti-EF-1 $\alpha$ from Upstate (Charlottesville, VA), anti-GATA-1 antibodies and goat anti-mouse IgGHRP from Santa Cruz Biotechnology (Santa Cruz, CA). Generation of rabbit anti-rat ALCAM antibody BRI-1 has previously been described as anti-HB2 $[39,40]$. BRI1 antiserum was purified on a protein-A column (ImmunoPure Plus High Capacity, Pierce Biotechnology, Inc. Rockford, IL).

\section{Cell culture}

K562 and HL-60 cells were cultured in Iscove's Modified Dulbecco's Medium (IMDM) (Invitrogen, Carlsbad, CA), supplemented with $10 \%$ fetal bovine serum (FBS) and $1 \%$ penicillin and streptomycin sulfate. THP-1, Jurkat, U-937 and MEG-01 cells were cultured in RPMI 1640 medium (ATCC, Manassas, VA) supplemented with $10 \% \mathrm{FBS}$. Cells were incubated at $37^{\circ} \mathrm{C}$ in a humidified chamber with $5 \% \mathrm{CO}_{2}$. For cell cluster formation 
analysis, single cell suspensions of K562-ALCAM $(2 \times$ $10^{5}$ ) were seeded in 24-well plates. Affinity purified BRI-1 antibody $(10 \mu \mathrm{g} / \mathrm{ml})$ or non-immune IgG or chelerythrine chloride $(10 \mu \mathrm{M})$ was added to cultures and examined 24 hours later. For disaggregation studies, K562-ALCAM cells $\left(2 \times 10^{5}\right)$ were allowed to form large aggregates over a 24 -hour period in 24-well plates, and then the cultures were gently treated with BRI-1 antibody $(10 \mu \mathrm{g} / \mathrm{ml})$ or a concentration range $(5-20 \mu \mathrm{M})$ of chelerythrine chloride. Cultures were photographed using Olympus IX50 microscope with digital image (Olympus Optical Co. Ltd. Japan).

\section{Western blot analysis}

Cell lysates prepared with ice-cold Cell Lysis Buffer (Cell Signaling Technology, Beverly, MA) containing 1\% triton $\mathrm{X}-100(\mathrm{v} / \mathrm{v})$ and supplemented with $1 \%$ protease inhibitor cocktail (Roche, Indianapolis, IN) was clarified by centrifugation at $13,000 \mathrm{rpm}$ for $15 \mathrm{~min}$ at $4^{\circ} \mathrm{C}$, and soluble cell fractions harvested. Protein content in cell lysates was measured using a Lowry protein assay (Sigma, St Louis, MO). Lysates were combined with buffer (Sigma, St Louis), boiled for 2 min and resolved by electrophoresis on a $10 \%$ polyacrylamide gel. Samples were blotted unto nitrocellulose membranes, probed with antibodies. The protein bands were identified by chemiluminescence (Fujifilm, Japan).

\section{Quantitative RT-PCR}

Total RNA was extracted from cultured cells using RNeasy Mini Kit (Qiagen, Valencia, CA), and converted to cDNA using SuperScript RT II (Invitrogen,). Quantitative RT-PCR was performed using gene-specific primers (Table 1), in reactions containing SYBR Green PCR master mixture (Applied Biosystems, Foster City, CA), on a StepOnePlus analyzer (Applied Biosystems). Relative expression level of target genes was normalized using GAPDH mRNA.

\section{Stable K562 cells expressing ALCAM}

We have previously described the cloning of rat ALCAM-GFP [19]. Super coiled plasmid DNA $(2.5 \mu \mathrm{g})$ of ALCAM-GFP or GFP was transfected into log-phase growing K562 cells using lipofectamine 2000 (Invitrogen). Stable lines were selected with G418 $(1200 \mu \mathrm{g} / \mathrm{ml})$ for 20 days. Expression of ALCAM-GFP and GFP was examined by epifluorescence (Nikon TE2000, Nikon Instruments Inc., Melville, NY).

\section{Reporter constructs and Site-directed mutagenesis}

Human genomic DNA was amplified by PCR using a common reverse primer complimentary to DNA sequence 60 base pairs (bp) upstream of the translation start site in the ALCAM gene, and various forward primers truncated at $-1200,-1000,-800,-650,-400$ and

Table 1 Sequence of Primers and DNA probes

\begin{tabular}{|c|c|c|}
\hline ALCAM Luc reporter constructs & Forward & Reverse \\
\hline-1200 & 5'-AAATCACCGCTTAACTCAAAG-3' & 5'-CCTCCTCCTTCTTGGTGG-3' \\
\hline-1000 & 5'-CCGCGCTTCAACCACCTGCT-3' & \\
\hline-800 & 5'-CAGAAAGTGTTAGTCCCAGG-3' & \\
\hline-650 & 5'-CCGCGCTTCAACCACCTGCT-3' & \\
\hline-400 & 5'-CCGCCTCCTGCGAGTCCTTC-3' & \\
\hline-200 & 5'-GTTGACCGGGAGGGAGGAGG-3' & \\
\hline EMSA probes & Sense strand & \\
\hline - 850 GATA-1 wt & 5'-GCCAGAGGCCTTATCACTGG-3' & \\
\hline - 850 GATA-1 mt & 5'-GCCAGAGGCCTGCTCACTGG-3' & \\
\hline ChIP assay & Forward & Reverse \\
\hline GATA-1 & 5'-GGGTGGAGGGAGAGGGCAGTC-3' & 5'-AAGCAAGCACAGGAGCCGCGC-3' \\
\hline RT-PCR & Forward & Reverse \\
\hline ALCAM & 5'-AGTCTT CAT TATCAGGATGC-3' & 5'-GGGATCAGTITTCTITGTCA-3' \\
\hline$\gamma$-globin & 5'-ACTCGCTTCTGGAACGTCTGA-3' & 5'-GTATCTGGAGGACAGGGCACT-3' \\
\hline Glycophorin A & 5'-GGAATTCCAGCTCATGATCTCAGGATG-3' & 5'-TCCACATTTGGTTTGGTGAACAGAT3' \\
\hline Cmpl & 5'-GCACTGTGATGCTITATGCAAC-3' & 5'-TGAACGGTITAGAGGATGAGGA-3' \\
\hline CD44 & 5'-GATCCACCCCAATTCCATCTGTGC-3' & 5'-AACCGCGAGAATCAAAGCCAAGGC-3' \\
\hline CD61 & 5'-TATAGCATTGGACGGAAGGC-3' & 5'-GACCTCATTGTTGAGGCAGG-3' \\
\hline GAPDH & 5'-ATGGGGAAGGTGAAGGTCGG-3' & 5'-GACGGTGCCATGGAATTTGC-3' \\
\hline $\mathrm{PKC} \alpha$ & 5'-CGAGGAAGGAAACATGGAACTCAG-3' & 5'-CCTGTCGGCAAGCATCACCTाT-3' \\
\hline $\mathrm{PKC} \varepsilon$ & 5'-TCAATGGCCTTCTTAAGATCAAAA-3' & 5'-CCTGAGAGATCGATGATCACATAC-3' \\
\hline PKC $\theta$ & 5'-CTCGTCAAAGAGTATGTCGAATCA-3' & 5'-AATTCATTCAGTCCTITGTGTCACTC-3' \\
\hline PKC $\delta$ & 5'-GCATCGCCTTCAACTCCTATGAGCT-3' & 5'-ACACACCCACGGTCACCTCAGA-3' \\
\hline
\end{tabular}


-200 (Table 1). These DNA fragments were then cloned into a promoter-less luciferase vector pGL3B (Promega, Madison, WI) via Kpn I and Sma I cleavage sites. The ALCAM-luciferase vectors (p1200ALCAMLuc, p1000ALCAMLuc, p800ALCAMLuc, p650ALCAMLuc, p400ALCAMLuc and p200ALCAMLuc) were generated and verified by sequence analysis. Two bp substitutions were generated in the GATA-1 binding site at -850 (CCTTATCACT $\rightarrow$ CCTGCTCACT) by site-directed mutagenesis (QuikChange, Stratagene, La Jolla, CA), and verified by DNA sequencing.

\section{Reporter gene assays}

Cells $\left(2 \times 10^{5}\right)$ were seeded in 24-well tissue culture plates and co-transfected with ALCAM promoter luciferase plasmids (800 ng) and pcDNA3.1/His/LacZ (100 ng) (Invitrogen) plasmid DNA using lipofectamine 2000. Twenty-four hours after transfection, cell lysates were prepared and the activities of luciferase (Firefly-Luciferase Reporter Assay System, Promega ) and $\beta$-galactosidase (Galacto-Star system, Applied Biosystems) were determined using the Veritas Luminometer (Turner Biosystems, Sunnyvale, CA). Luciferase activity was normalized to the activity of $\beta$-galactosidase, and the relative luciferase activity for test constructs calculated by assigning the normalized luciferase activity of the promoter-less pGL3 construct as 1.0.

\section{Electrophoretic mobility shift assay (EMSA)}

In vitro protein-DNA interaction was examined using the LightShift Chemiluminescence electrophoretic mobility shift assay (EMSA) kit (Pierce, Rockford, IL). ALCAMspecific EMSA DNA probes were synthesized, gel purified and biotin labeled (Table 1) (Integrated DNA Technologies, Coralville, IA). Nuclear extract $(4 \mu \mathrm{g})$ was combined with biotin-labeled DNA probes in binding buffer containing $50 \mu \mathrm{g} / \mathrm{ml}$ poly (dI-dC). Molar excess (50-200-fold) of unlabelled DNA probe or anti-GATA-1 antibody $(2 \mu \mathrm{g})$ (Santa Cruz Biotechnology) was added to the binding reaction in competition experiments. Products of the binding reaction were resolved in 6\% DNA retardation gel (Invitrogen), transferred to a nylon membrane and biotinlabeled complexes detected by chemiluminescence (Fujifilm LAS-1000 imaging system, Valhalla, NY).

\section{Chromatin immunoprecipitation assay (ChIP)}

For ChIP assay, protein-DNA cross-linking was performed by fixing 40 million cells with $1 \%$ formaldehyde. Nuclei were sonicated on ice in shearing buffer (ChIPIT, Active Motif, Carlsbad, CA) to obtain chromatin fragments of 1000-100 base pairs, which were precleared with protein G beads (Salmon sperm DNA/Protein $\mathrm{G}$ agarose). Alternatively, pre-cleared chromatin was incubated with anti-GATA-1 (Santa Cruz Biotechnology), anti-GAPD or non-immune IgG. Immune complexes were precipitated with protein $\mathrm{G}$ beads, and the eluate reversed cross-linked in $190 \mathrm{mM} \mathrm{NaCl}$. DNA was purified by centrifugation on a mini-column and amplified by PCR with specific ALCAM primers (Table 1). PCR products were resolved by electrophoresis in $2 \%$ agarose gels, visualized by ultraviolet illumination and digital images recorded using GeneFlash gel documentation system (SynGene, Frederick, MD).

\section{Apoptosis and TUNEL assays}

Cells $\left(1 \times 10^{6}\right)$ were plated in 6-well plates and incubated with PMA (20 nM) or vehicle (DMSO). Twentyfour hours later, cells were stained with phycoerythrin (PE) labeled annexin $\mathrm{V}$ and 7-Amino-actinomycin (7-ADD), using the Apoptosis Detection kit (BD Biosciences, San Jose, CA). Cells were then analyzed by flow cytometry (FACScan Advantage, BD Biosciences) and data from 10,000 events collected for analysis. For the TUNEL (terminal deoxynucleotidyl transferasemediated deoxyuridine triphosphate nick end labeling) assay, cells $\left(1 \times 10^{6}\right)$ were plated in 6 -well plates, and treated for 48 hours with PMA $(20 \mathrm{nM})$ or vehicle (DMSO). Cells were fixed using freshly diluted $1 \%$ paraformaldehyde in PBS for 10 minutes at room temp. Cell monolayers were prepared by cytospin on a silanized microscope slide, apoptotic cells were identified using the in situ ApopTag Peroxidase kit (Chemicon International, Temecula, CA). Methyl green solution $(0.5 \%)$ was used to counter-stain the slides. Apoptotic cells were counted in 10 random fields using $20 \times$ microscope objective and the percentage TUNEL-positive cells calculated.

\section{Statistics}

Data were analyzed using GraphPad Prism software (version 5 ). Student's $t$-test was used to measure differences in samples of two groups. Probability of less than 0.05 was considered statistically significant. Levels of significance are: $\left.\mathrm{p}<0.05{ }^{(*)}\right), \mathrm{p}<0.01{ }^{(* *)}$ and $\mathrm{p}<0.001{ }^{(* * *)}$.

\section{List of abbreviations used}

ALCAM: activated leukocyte cell adhesion molecule; RACE: rapid amplification of CDNA ends; EMSA: Electrophoretic mobility shift assay; CHiP: Chromatin immunoprecipitation assay.

\section{Acknowledgements}

This work was supported by grants R01HL077769 awarded to Solomon F Ofori-Acquah. We are grateful to Dr. R Fillmore for guidance with the ChIP assay and Ms. R Cochran for technical help.

\section{Author details}

'Department of Pediatrics, Aflac Cancer Center and Blood Disorders Service, Emory University School of Medicine, Atlanta, GA 30322, USA. ${ }^{2}$ Department 
of Cell Biology and Neuroscience, University of South Alabama, Mobile, AL 36688, USA. ${ }^{3}$ Department of Biology, The University of lowa, lowa City, IA 52242, USA.

\section{Authors' contributions}

FT performed experiments and prepared the manuscript. SG, FM and RT performed experiments, JAW provided ALCAM null mice and SFOA conceived of the study, interpreted data and wrote the manuscript. All authors read and approved the final manuscript.

Received: 10 September 2010 Accepted: 2 December 2010

Published: 2 December 2010

\section{References}

1. Yin T, Li L: The stem cell niches in bone. The Journal of clinical investigation 2006, 116(5):1195-1201.

2. Li L, Xie T: Stem cell niche: structure and function. Annual review of cell and developmental biology 2005, 21:605-631.

3. Nilsson SK, Johnston HM, Whitty GA, Williams B, Webb RJ, Denhardt DT, Bertoncello I, Bendall LJ, Simmons PJ, Haylock DN: Osteopontin, a key component of the hematopoietic stem cell niche and regulator of primitive hematopoietic progenitor cells. Blood 2005, 106(4):1232-1239.

4. Stier S, Ko Y, Forkert R, Lutz C, Neuhaus T, Grunewald E, Cheng T, Dombkowski D, Calvi LM, Rittling SR, et al: Osteopontin is a hematopoietic stem cell niche component that negatively regulates stem cell pool size. $J$ Exp Med 2005, 201(11):1781-1791.

5. Quesenberry PJ, Becker PS: Stem cell homing: rolling, crawling, and nesting. Proc Natl Acad Sci USA 1998, 95(26):15155-15157.

6. Hirsch E, Iglesias A, Potocnik AJ, Hartmann U, Fassler R: Impaired migration but not differentiation of haematopoietic stem cells in the absence of beta1 integrins. Nature 1996, 380(6570):171-175.

7. Potocnik AJ, Brakebusch C, Fassler R: Fetal and adult hematopoietic stem cells require beta1 integrin function for colonizing fetal liver, spleen, and bone marrow. Immunity 2000, 12(6):653-663.

8. Zhang J, Niu C, Ye L, Huang H, He X, Tong WG, Ross J, Haug J, Johnson T, Feng $\mathrm{JQ}$, et al: Identification of the haematopoietic stem cell niche and control of the niche size. Nature 2003, 425(6960):836-841.

9. Wilson A, Murphy MJ, Oskarsson T, Kaloulis K, Bettess MD, Oser GM, Pasche AC, Knabenhans C, Macdonald HR, Trumpp A: c-Myc controls the balance between hematopoietic stem cell self-renewal and differentiation. Genes \& development 2004, 18(22):2747-2763.

10. Haug JS, He XC, Grindley JC, Wunderlich JP, Gaudenz K, Ross JT, Paulson A, Wagner KP, Xie $Y$, Zhu R, et al: N-cadherin expression level distinguishes reserved versus primed states of hematopoietic stem cells. Cell stem cell 2008, 2(4):367-379.

11. Kiel MJ, Acar M, Radice GL, Morrison SJ: Hematopoietic stem cells do not depend on $\mathrm{N}$-cadherin to regulate their maintenance. Cell stem cell 2009, 4(2):170-179.

12. Uchida N, Yang Z, Combs J, Pourquie O, Nguyen M, Ramanathan R, Fu J, Welply A, Chen S, Weddell G, et al: The characterization, molecular cloning, and expression of a novel hematopoietic cell antigen from CD34+ human bone marrow cells. Blood 1997, 89(8):2706-2716.

13. Cortes F, Deschaseaux F, Uchida N, Labastie MC, Friera AM, He D, Charbord $P$, Peault B: HCA, an immunoglobulin-like adhesion molecule present on the earliest human hematopoietic precursor cells, is also expressed by stromal cells in blood-forming tissues. Blood 1999, 93(3):826-837.

14. Stephan JP, Bald L, Roberts PE, Lee J, Gu Q, Mather JP: Distribution and function of the adhesion molecule BEN during rat development. Dev Biol 1999, 212(2):264-277

15. Ohneda O, Ohneda K, Arai F, Lee J, Miyamoto T, Fukushima Y, Dowbenko D, Lasky LA, Suda T: ALCAM (CD166): its role in hematopoietic and endothelial development. Blood 2001, 98(7):2134-2142.

16. Bowen MA, Bajorath J, Siadak AW, Modrell B, Malacko AR, Marquardt H, Nadler SG, Aruffo A: The amino-terminal immunoglobulin-like domain of activated leukocyte cell adhesion molecule binds specifically to the membrane-proximal scavenger receptor cysteine-rich domain of CD6 with a 1:1 stoichiometry. J Biol Chem 1996, 271(29):17390-17396.

17. Degen WG, van Kempen LC, Gijzen EG, van Groningen JJ, van Kooyk Y, Bloemers HP, Swart GW: MEMD, a new cell adhesion molecule in metastasizing human melanoma cell lines, is identical to ALCAM (activated leukocyte cell adhesion molecule). Am J Pathol 1998, 152(3):805-813.

18. Corbel C, Pourquie O, Cormier F, Vaigot P, Le Douarin NM: BEN/SC1/DMGRASP, a homophilic adhesion molecule, is required for in vitro myeloid colony formation by avian hemopoietic progenitors. Proc Natl Acad Sci USA 1996, 93(7):2844-2849.

19. Masedunskas A, King JA, Tan F, Cochran R, Stevens T, Sviridov D, OforiAcquah SF: Activated leukocyte cell adhesion molecule is a component of the endothelial junction involved in transendothelial monocyte migration. FEBS letters 2006, 580(11):2637-2645.

20. Cayrol R, Wosik K, Berard JL, Dodelet-Devillers A, Ifergan I, Kebir H, Haqqani AS, Kreymborg K, Krug S, Moumdjian R, et al: Activated leukocyte cell adhesion molecule promotes leukocyte trafficking into the central nervous system. Nature immunology 2008, 9(2):137-145.

21. Zimmerman AW, Joosten B, Torensma R, Parnes JR, van Leeuwen FN, Figdor CG: Long-term engagement of CD6 and ALCAM is essential for Tcell proliferation induced by dendritic cells. Blood 2006, 107(8):3212-3220.

22. Goldfarb AN, Delehanty LL, Wang D, Racke FK, Hussaini IM: Stromal inhibition of megakaryocytic differentiation correlates with blockade of signaling by protein kinase C-epsilon and ERK/MAPK. J Biol Chem 2001, 276(31):29526-29530

23. Racke FK, Wang D, Zaidi Z, Kelley J, Visvader J, Soh JW, Goldfarb AN: A potential role for protein kinase C-epsilon in regulating megakaryocytic lineage commitment. J Biol Chem 2001, 276(1):522-528.

24. Corbel C, Cormier F, Pourquie O, Bluestein HG: BEN, a novel surface molecule of the immunoglobulin superfamily on avian hemopoietic progenitor cells shared with neural cells. Exp Cell Res 1992, 203(1):91-99.

25. Simon MC, Pevny L, Wiles MV, Keller G, Costantini F, Orkin SH: Rescue of erythroid development in gene targeted GATA-1-mouse embryonic stem cells. Nat Genet 1992, 1(2):92-98.

26. Pevny L, Lin CS, D'Agati V, Simon MC, Orkin SH, Costantini F: Development of hematopoietic cells lacking transcription factor GATA-1. Development 1995, 121(1):163-172.

27. Raich N, Clegg CH, Grofti J, Romeo PH, Stamatoyannopoulos G: GATA1 and YY1 are developmental repressors of the human epsilon-globin gene. EMBO J 1995, 14(4):801-809.

28. Rincon-Arano H, Valadez-Graham V, Guerrero G, Escamilla-Del-Arenal M, Recillas-Targa F: YY1 and GATA-1 interaction modulate the chicken 3'side alpha-globin enhancer activity. J Mol Biol 2005, 349(5):961-975.

29. Pevny L, Simon MC, Robertson E, Klein WH, Tsai SF, D'Agati V, Orkin SH, Costantini F: Erythroid differentiation in chimaeric mice blocked by a targeted mutation in the gene for transcription factor GATA-1. Nature 1991, 349(6306):257-260.

30. Jezierska A, Matysiak W, Motyl T: ALCAM/CD166 protects breast cancer cells against apoptosis and autophagy. Med Sci Monit 2006, 12(8): BR263-273.

31. Swart GW, Lunter PC, Kilsdonk JW, Kempen LC: Activated leukocyte cell adhesion molecule (ALCAM/CD166): signaling at the divide of melanoma cell clustering and cell migration? Cancer Metastasis Rev 2005, 24(2):223-236

32. Gobbi G, Mirandola P, Sponzilli I, Micheloni C, Malinverno C, Cocco L, Vitale M: Timing and expression level of protein kinase $C$ epsilon regulate the megakaryocytic differentiation of human CD34 cells. Stem Cells 2007, 25(9):2322-2329.

33. Lee JY, Hannun YA, Obeid LM: Ceramide inactivates cellular protein kinase Calpha. J Biol Chem 1996, 271(22):13169-13174.

34. Goss VL, Hocevar BA, Thompson LJ, Stratton CA, Burns DJ, Fields AP: Identification of nuclear beta II protein kinase $C$ as a mitotic lamin kinase. J Biol Chem 1994, 269(29):19074-19080.

35. Datta R, Kojima H, Yoshida K, Kufe D: Caspase-3-mediated cleavage of protein kinase $C$ theta in induction of apoptosis. J Biol Chem 1997, 272(33):20317-20320.

36. Schultz A, Jonsson Jl, Larsson C: The regulatory domain of protein kinase Ctheta localises to the Golgi complex and induces apoptosis in neuroblastoma and Jurkat cells. Cell Death Differ 2003, 10(6):662-675.

37. Sivaprasad U, Shankar E, Basu A: Downregulation of Bid is associated with PKCepsilon-mediated TRAIL resistance. Cell Death Differ 2007, 14(4):851-860.

38. Steinberg R, Harari OA, Lidington EA, Boyle JJ, Nohadani M, Samarel AM, Ohba M, Haskard DO, Mason JC: A protein kinase Cepsilon-anti-apoptotic kinase signaling complex protects human vascular endothelial cells 
against apoptosis through induction of Bcl-2. J Biol Chem 2007, 282(44):32288-32297.

39. Matsumoto A, Mitchell A, Kurata $H$, Pyle L, Kondo K, Itakura H, Fidge N: Cloning and characterization of HB2, a candidate high density lipoprotein receptor. Sequence homology with members of the immunoglobulin superfamily of membrane proteins. J Biol Chem 1997, 272(27):16778-16782

40. Sviridov D, Sasahara T, Pyle LE, Nestel PJ, Fidge NH: Antibodies against high-density lipoprotein binding proteins enhance high-density lipoprotein uptake but do not affect cholesterol efflux from rat hepatoma cells. Int J Biochem Cell Biol 1997, 29(4):583-588.

doi:10.1186/1471-2199-11-91

Cite this article as: Tan et al:: Essential role for ALCAM gene silencing in megakaryocytic differentiation of K562 cells. BMC Molecular Biology 2010 11:91.

\section{Submit your next manuscript to BioMed Central} and take full advantage of:

- Convenient online submission

- Thorough peer review

- No space constraints or color figure charges

- Immediate publication on acceptance

- Inclusion in PubMed, CAS, Scopus and Google Scholar

- Research which is freely available for redistribution

Submit your manuscript at www.biomedcentral.com/submit
C Biomed Central 